\title{
Mechanical and neural responses from the mechanosensory hairs on the antennule of Gaussia princeps
}

\author{
D. M. Fields*, D. S. Shaeffer, M. J. Weissburg \\ Georgia Institute of Technology, School of Biology, 310 Ferst Drive, Atlanta, Georgia 30332, USA
}

\begin{abstract}
This study investigated the physical and physiological response of individual setae on the antennule of Gaussia princeps. We found significant differences in the physical and physiological responses of the setae to various intensities of water flow. No physiological evidence was found to suggest that individual setae are dually innervated; however, directional bias in both the displacement and subsequent physiological responses was evident. Although more easily displaced by fluid flow, the shortest hairs were physiologically less sensitive to angular deflection than were the longer setae, so that slow flows produced a greater neural response in the long seta. The combination of high resistance to movement and acute physiological sensitivity allows the long seta to respond to biologically driven, low-intensity flows while filtering out high-frequency background noise. This suggests that the most prominent, long, distal setae function as low-flow detectors whereas the short hairs respond to more rapid fluid motion. Each seta responds to only a portion of the overall range of water velocity in the copepod's habitat. Thus, the entire sensory appendage, which consists of an ensemble of setae of different morphologies and lengths, may function as a unit to code the intensity and directionality of complex fluid disturbances.
\end{abstract}

KEY WORDS: Crustacean · Mechanoreception · Neurophysiology · Sensory perception · Fluid mechanical signals

Resale or republication not permitted without written consent of the publisher

\section{INTRODUCTION}

The behavior of pelagic copepods is not random. Numerous studies have shown that copepods respond remotely to potential mates (Doall et al. 1998, Weissburg et al. 1998), predators (Drenner \& McCormas 1980), and prey (Siaz \& Kiørboe 1995, Jonsson \& Tiselius 1990). Although the stimuli used to identify and locate other individuals may involve a cocktail of chemical and fluid mechanical signals, the results of previous studies have shown that mechanical signals are sufficient to elicit feeding (Yen \& Fields unpubl. data) and escape reactions (Haury et al. 1980, Fields \& Yen 1997, Kiørboe et al. 1999), and are likely to be

*E-mail: david.fields@biology.gatech.edu important for the final steps of mate capture (Doall et al. 1998). These observations suggest that fluid mechanical signals convey information on the identity as well as the location of the signal source perceived by zooplankton.

The implicit assumptions of studying animal behavior are that organisms can discern different signals and that the uniqueness of the signals is the basis for their response. Interpreting mechanosensory behavior of copepods therefore requires an understanding of the characteristics of the fluid signal, the physical and physiological response of the sensor to the fluid signal, and the relationship between the physiology and animal behavior.

Only recently have visualization techniques become sophisticated enough to map the complex flow fields produced by moving animals. Locomoting fish and 
large benthic crustaceans produce fluid disturbances with a power spectra characterized by strong frequencies below $10 \mathrm{~Hz}$, although some higher frequency components (25 to $200 \mathrm{~Hz}$ ) are present in vortices shed from the caudal fins of fish (Breithaupt \& Ayers 1996). Fluid disturbances created by swimming copepods produce spatially variable velocity profiles with typical deformation rates of 5 to $10 \mathrm{~s}^{-1}$ (Fields \& Yen 1993, unpubl. data) and frequencies between 10 and $50 \mathrm{~Hz}$. During rapid escape reactions, copepods release small vorticles of 1 to $2 \mathrm{~cm}$ diameter at frequencies of 30 to $100 \mathrm{~Hz}$ (Yen \& Strickler 1996). Based on direct force measurements from escaping copepods, pulses derived from independent movement of the pereiopods can achieve frequencies in excess of $500 \mathrm{~Hz}$ in the near-field (Lenz \& Hartline 1999). While the behavioral responses to these types of fluid disturbances has received some attention (Fields \& Yen 1996, 1997, Yen \& Strickler 1996), far less is known about the physiological properties of copepod mechanosensors.

One of the distinctive sensory organs of calanoid copepods is a pair of long antennules adorned with both mechano- and chemoreceptors. Feeding or swimming individuals create a flow field that passes fluid over the sensory array on the antennules. Near the head of the copepod, the antennules experience the maximum flow rate of the feeding current (Fields \& Yen 1993). In contrast, because of the length of the antennules, the distal tips often extend outside the fluid disturbance created by the copepod's own flow field and expose it to the relatively quiescent regions of the surrounding fluid (Fields \& Yen 1993). Recent data suggest that this region of the antennule may serve as the primary location for the detection of much larger feeding currents or fluid disturbances created by potential predators (Lenz \& Yen 1993). Physiological data have shown that setae in the distal region are extremely sensitive to water motion, with neural activity in response to setal tip displacements of less than $10 \mathrm{~nm}$ (Yen et al. 1992). However, neural stimulation is not synonymous with stimulus recognition. The observed behavior patterns require that the copepods be able to differentiate complex fluid signals in order to respond in an appropriate manner.

One mechanism contributing to stimulus recognition is for different sensors to be preferentially tuned to detect particular types of signals. Many different sensillum types occupy copepod antennules (Friedman \& Strickler 1975, Strickler \& Bal 1973, Gill \& Crisp 1985, Bundy \& Paffenhöfer 1993, Weatherby et al. 1994). For example, within the final 2 antennule segments of Pleuromamma xiphias, 8 different setal morphologies have been characterized (Weatherby et al. 1994). Difference in sensor morphology is likely to be fundamental to the copepod's ability to detect complex fluid structures containing spatially variable velocity components; yet, despite evidence for the critical role of mechanoreception in the ecology of copepods, little is known about the comparative sensitivity of different setae along their antennules.

In an effort to address the underlying mechanism of how differences in setal properties affect the ability of copepods to decode fluid mechanical signals, we designed experiments that allow the simultaneous quantification of stimulus strength at the sensor and the sensor's mechanical and physiological response. Experiments were conducted on different setae within the distal region of the antennule of Gaussia princeps. By selecting setae with different morphologies, we could compare the coupling of physical and physiological responses to water motion of different setae types.

\section{MATERIALS AND METHODS}

Collection and maintenance. Gaussia princeps is a large (8 mm), bioluminescent (Buskey et al. 1994), carnivorous copepod that resides at depth greater than $500 \mathrm{~m}$. Individuals were captured from the RV 'Pt. Sur' operated by the Monterey Bay Aquarium Research Institute (MBARI). Midwater trawls were taken at depths of 600 to $800 \mathrm{~m}$ where the water temperature was approximately $3.9^{\circ} \mathrm{C}$. The copepods were transported by air (in ice-filled containers that maintained temperatures of 4 to $6^{\circ} \mathrm{C}$ ) to our laboratory at Georgia Institute of Technology (GIT). At GIT, they were kept in the dark at $4^{\circ} \mathrm{C}$ and maintained in concentrations of 5 copepods $\mathrm{l}^{-1}$ on a diet of freshly hatched Artemia nauplii for up to $3 \mathrm{wk}$.

Experimental setup. The experimental apparatus consists of: (1) an optical pathway to record setal motion (filmed using standard video); (2) a stimulus (water jetfilmed using high-speed video, $2000 \mathrm{~Hz}$ ); (3) a system to extracellularly record nerve depolarization (Fig. 1). The timing of the stimulus and the collection of physiological data were coupled through an externally generated TTL (transmitter-transmitter logic ; 5 V electronic signal) pulse with a timing accuracy of $10 \mu \mathrm{s}$. Flow visualization of the fluid disturbance created by the water jet was accomplished using a Schlieren optical pathway and a high-speed video-recording system. By amalgamating neurophysiological with video techniques, we were able to couple ambient fluid motion to the physical motion of individual mechanosensors (seta). The motion of the seta could then be correlated to its neurophysiology, allowing us to trace the entire transduction pathway from fluid disturbance to sensory response.

Recording setal motion: Setal bending was analyzed from SVHS (Panasonic AG 1970) video-recordings, filmed using a Pulnix TM 745 CCD camera mounted 
Fig. 1. Schematic of the experimental apparatus. The technique includes 3 technologies: a video-recording (60 or $2000 \mathrm{~Hz}$ ) device, a technique to measure physiology from the antennule of copepods, and a mechanism to deliver a controlled fluid mechanical signal. TTL: transmitter-transmitter logic (+5 V signal)

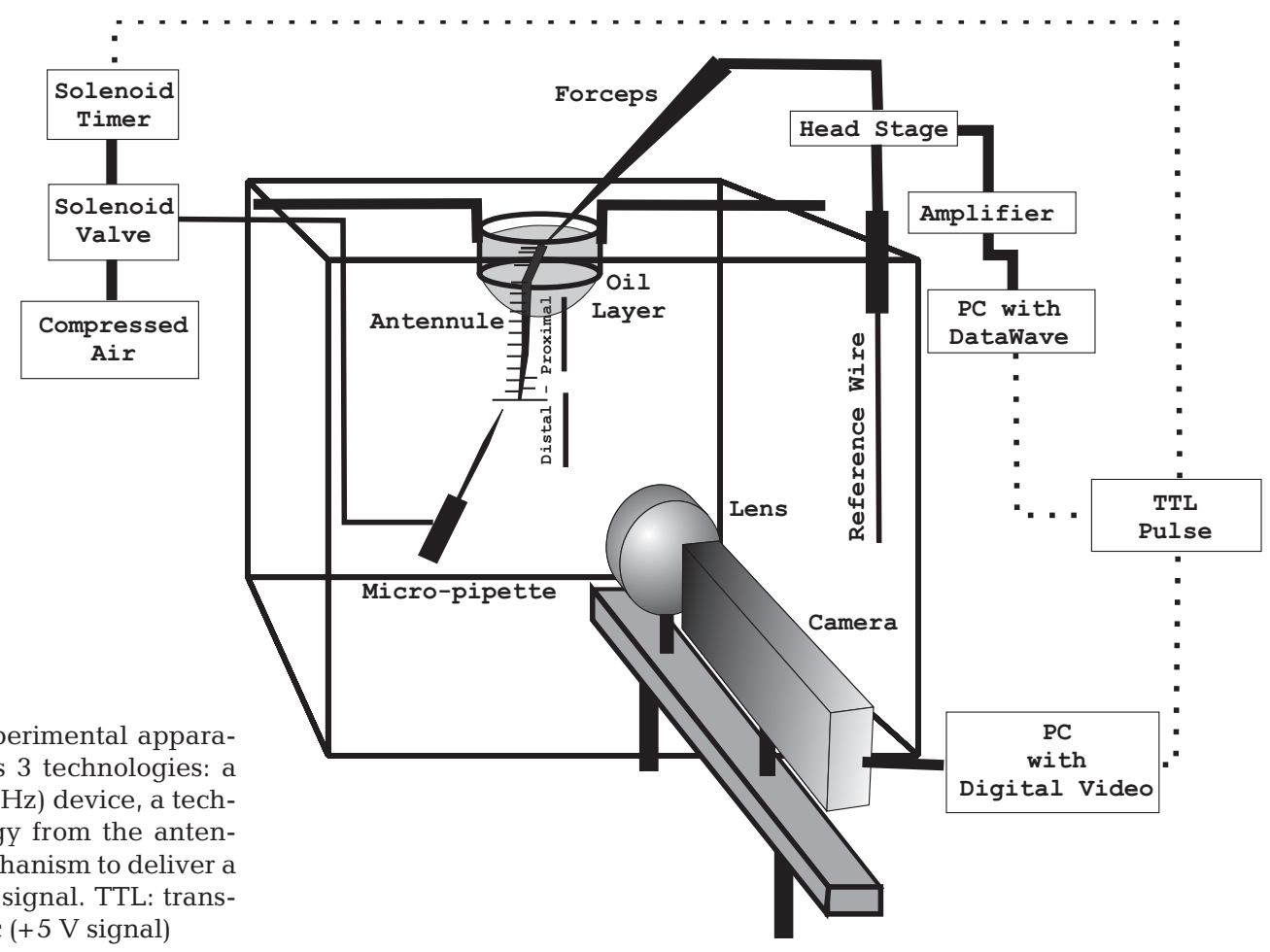

in a bellow style with a $105 \mathrm{~mm}$ macro Nikon lens. Camera shutter speed was set at 1/500th to 1/1000th $\mathrm{s}$ to minimize blurring of the seta within one frame of video. Individual fields of video were time-stamped with a Horita TRG-50 time-code generator. Illumination was achieved using 2 fiber-optic lights with 1 positioned parallel to and the other perpendicular to the camera. Video-analysis was accomplished at a resolution of $60 \mathrm{~Hz}$ by splitting the video frame into 2 component fields using an analog-to-digital video-recorder (PVR Digital Processing Systems).

The degree of setal bending was quantified from video-recordings using NIH Image (Scion Corporation). The coordinates of 3 points along the seta (base, midpoint, and tip of the seta) and 1 point on the antennule were collected prior to bending to define the angle of the seta with respect to the antennule (reference angle). Coordinates were subsequently collected for each frame of the bend cycle. Displacement was calculated using the law of cosines. At high stimulus intensity there was considerable difference in the degree of angular displacement at the tip versus near the base of the seta of Setae \#6 and \#7 (see Fig. 4). In this study we report the displacement that occurred near the base of the seta. For each copepod, bending was recorded for 5 trials at each experimental condition, and the average maximum angular displacement was calculated for each condition.

Water jet: The following procedure and calibration were used.
Procedure: A small water jet was used to stimulate individual seta. The water jet was created using a $1 \mathrm{~mm}$ outer diameter (o.d.) capillary tube stetched to an o.d. of $150 \mu \mathrm{m}$ and an inner diameter (i.d.) of $75 \mu \mathrm{m}$ at the tip. The pipette was filled with ambient fluid and was then expelled forcibly by compressed air at a pressure of $344 \mathrm{kPa}$ (50 psi) using a micro-injection system (Picospritzer II). The speed of the water jet was controlled by the length of time that the solenoid valve remained open. The size and precision of the jet allowed stimulation of individual setae, and previous work has shown that fluid disturbances created in this manner mimic the natural fluid disturbance created by swimming and escaping copepods (Yen 2000, Yen \& Fields unpubl. data).

Calibration: The characteristics of the water jet were quantified using a Schlieren optical pathway. Filming was done using a single low-powered He-Ne laser (5 mW-632 nm, Newport Corporation). The $0.8 \mathrm{~mm}$ beam was split, expanded to $20 \mathrm{~mm}$, and then used to illuminate 2 perpendicularly mounted video cameras. To measure the characteristics of the expelled fluid, we loaded the pipette with 28 ppt salt water and ejected it into our experimental vessel filled with 34 ppt salt water. Although there was only a slight difference in fluid density $(0.6 \%)$, the different fluids provided enough optical difference to be detected using our Schlieren pathway. Fluid motion was filmed digitally at $2000 \mathrm{~Hz}$ and an equivalent shutter speed of 1/40 $000 \mathrm{~s}$ 
(Motion Scope PCI 2000s). Changes in the displacement of the head of the water jet over time were quantified from sequential video images using NIH Image software. The data was used to calculate fluid displacement and speed. The data were fit to a Weibull Peak equation (4 parameter) to estimate the fluid velocity at the specific distance of the setae for each experiment.

Neurophysiology: In preliminary experiments we found that amputated antennules gave recordings similar to whole-copepod preparations. The amputated antennule was preferred, since it allowed us to individually test each antennule and because the antennule could be positioned much more easily than the entire copepod. The antennule was ablated between the 2nd and 5th segments, and neural recordings were done following the technique described by Gassie et al. (1993). Briefly, the antennule was grasped at the base with stainless steel forceps and lowered into the experimental vessel through a $13 \mathrm{~mm} \times 20 \mathrm{~mm}$ (depth $\times$ diameter) Plexiglas tube. The Plexiglas tube, which was suspended just above the water surface, was filled with mineral oil (4 ml) until all the water was displaced from the tube. The forceps and the first 8 to $10 \mathrm{seg}$ ments of the antennule were then drawn out of the seawater into the electrically insulated oil layer. The electric potential was measured between the forceps and a silver reference wire (placed in the seawater) using standard extracellular recording techniques. This technique allows the detection of all the neural traffic that passes the oil-water interface travelling between the distal and proximal regions of the antennule. Neural responses were sorted based on their waveform characteristics (i.e. amplitude, rise time, offset slope) using commercially available software (Datawave; Longmont, Colorado) and matched to the waveform library created by the tactile stimulation (see following description).

General procedure and analysis. Individual setae were stimulated in 2 ways. First the seta received tactile stimulation in both the proximal and distal direction to survey nerves that signaled above background noise and to generate an experiment-specific library of the waveform of individual nerves associated with each seta. Once the library had been created, the mouth of the pipette was aimed normal to the seta and discharged for a duration of 1 to $30 \mathrm{~ms}$ in an incremental fashion. The pipette tip was positioned at a distance ranging from 150 to $800 \mu \mathrm{m}$ from the seta. Water speed was taken from calibration curves as discussed in the subsection 'Water jet'. Neural data were collected for a total of $100 \mathrm{~ms}$ prior to stimulation to determine the rate of spontaneous activity, and $1.4 \mathrm{~s}$ post stimulation. Physiological response to the water jet was calculated as the number of spikes during the first $200 \mathrm{~ms}$ post- stimulation corrected for spontaneous activity. Each duration was tested 5 times, with a minimum interstimulus period of $30 \mathrm{~s}$, and then averaged. For comparisons between different setae or between proximal verses distal stimulation of the same seta, the data were normalized to their individual maxima and fit to a Hill 3-parameter model as:

$$
\% \text { Spike }_{\max }=\frac{a x^{b}}{\left(c^{b}+x^{b}\right)}
$$

where $x$ is the independent variable (angular displacement or water jet speed), and $a, b, c$ are parameters solved in the equation.

\section{RESULTS}

\section{Water jet characteristics}

The water jet exits from the mouth of the pipette 9 to $20 \mathrm{~ms}$ after being triggered and forms an axisymmetric vortex that maintains a similar structure for over $3 \mathrm{~mm}$ from the pipette (Fig. 2). After opening the solenoid valve, the water remained stationary for 9 to $20 \mathrm{~ms}$ in the pipette and then accelerated as it exited the mouth (Fig. 3A). Thus, the head speed of the water jet increased to its maximum at some distance from the pipette tip (Fig. 3B). The water jet subsequently decreased in speed due to the interaction with the stationary ambient fluid (Fig. 3B: 5 and $9 \mathrm{~ms}$ ). The distance and speed that the expelled

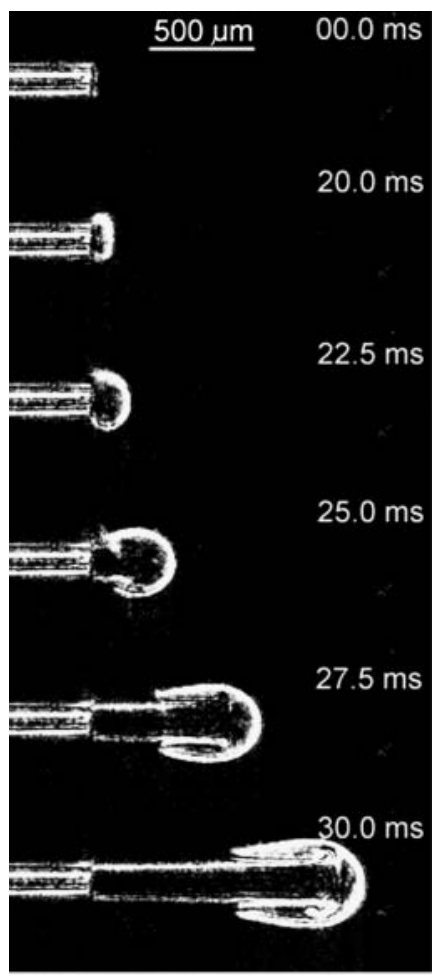

Fig. 2. Time series of the water jet released from a $150 \mu \mathrm{m}$ pipette. The pressure behind the solenoid was $344 \mathrm{kPa}$ and the solenoid remained open for $13 \mathrm{~ms}$. The movement of the expelled water was filmed at $2000 \mathrm{~Hz}$ using a Schlieren optical pathway. Note that the water only begins to exit the mouth of the pipette 16 to $20 \mathrm{~ms}$ after the solenoid is opened 

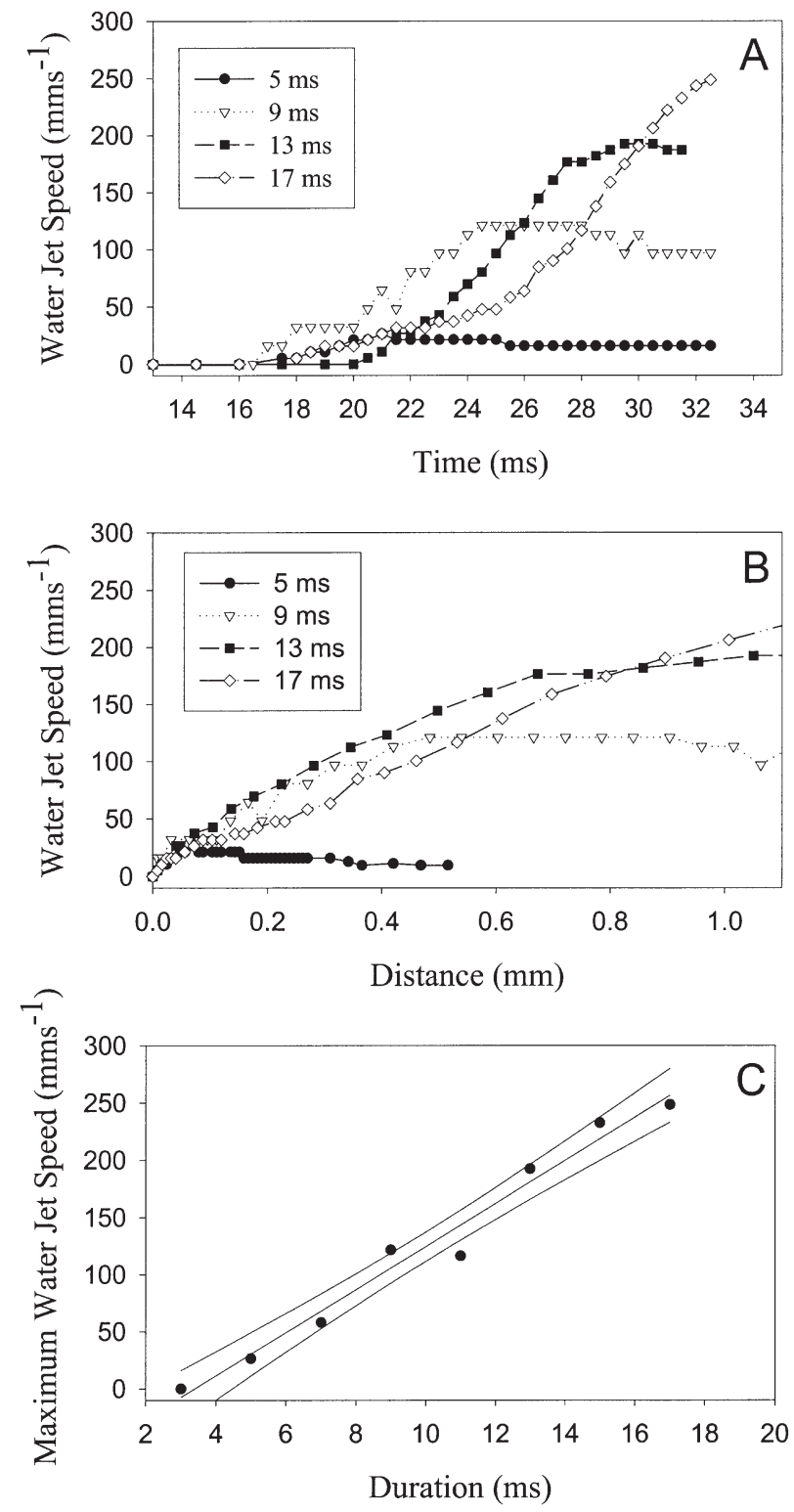

Fig. 3. Characterization of the fluid mechanical signal. (A) Speed of the water jet as a function of time after the solenoid was opened (time $=0 \mathrm{~ms}$ ); different symbols represent the different durations that the solenoid remained open; note that the water does not begin to exit the mouth of the pipette until $\sim 16$ to $20 \mathrm{~ms}$ after the solenoid is opened. (B) Speed of the water jet as a function of distance traveled from the pipette tip. (C) Maximum head speed of the water jet as a function of the duration that the solenoid remained open: data was fit to a linear regression with the following parameters $\left(\max\right.$. speed $=-36.8 \times$ duration $\left.+15.6 ; \mathrm{r}^{2}=0.97\right)$; top and bottom curves represent $95 \%$ confidence interval

water jet traveled were significantly correlated with the duration that the solenoid remained open $(p<0.001$; Fig. 3A,B). The maximum head speed of the ejected fluid was accurately predicted by the duration of time that the solenoid remained open (Fig. 3C).

\section{Physical response of the sensor}

The distal region of the antennule of Gaussia princeps bears setae of various lengths (Fig. 4, Table 1). When exposed to the water jet, setae display a characteristic angular displacement (Fig. 5). Setal angular displacement and angular velocity increased with increasing water jet speed (Fig. 6). However, there was a significant difference between the angular motion of the setae and the speed of the water. For example, at water jet speeds of $200 \mathrm{~mm} \mathrm{~s}^{-1}$ Seta \#8 moved at an angular speed of $120^{\circ} \mathrm{s}^{-1}$ (Fig. 6). At this water jet speed, the seta reached a maximum angular displacement of $3^{\circ}$, which corresponds to a tip displacement of $157 \mu \mathrm{m}$ for the $3 \mathrm{~mm}$ seta. Since the angular displacement occurred within $33 \mathrm{~ms}$, the seta must have traveled at an average speed of $4.8 \mathrm{~mm} \mathrm{~s}^{-1}$ or approximately $2 \%$ of the maximum fluid speed. The same calculation on Seta \#7 when displaced proximally shows that the $890 \mu \mathrm{m}$ seta moved $289 \mu \mathrm{m}\left(18^{\circ}\right)$ in $15 \mathrm{~ms}\left(1200^{\circ} \mathrm{s}^{-1}\right)$, which is equivalent to $8 \%$ of the maximum fluid motion. This suggests that, in general, the seta of G. princeps have considerable resistance to motion, with the longest seta (\#8) being less sensitive to fluid motion than the shorter seta (\#6 and \#7) (Fig. 7, Table 2).

Setal morphology also affected the response of the seta to directional stimulation. Given the same water speed, Seta \#7 achieved a smaller maximum angular displacement when stimulated proximally verses distally. Angular displacement in the distal direction was approximately 2 times greater than in the proximal direction at the same water speed. In contrast, Seta \#8 showed no significant difference in its physical response to directional stimulation, indicating that not all setae show a directional bias.

\section{Physiological response of the sensor}

Neural activity was recorded from 3 different setae in the distal region of the antennae of Gaussia prin-

Table 1. Gaussia princeps. Measurements (mean + SD) of the setae along the antennae. Seta identified as in Fig. 4. $\mathrm{n}$ : number of seta measured

\begin{tabular}{|lrr|}
\hline Seta & Length $(\mu \mathrm{m})$ & $\mathrm{n}$ \\
\hline 1 & $2039(86)$ & 2 \\
2 & $220(40)$ & 8 \\
3 & $223(35)$ & 6 \\
4 & $811(84)$ & 3 \\
5 & $409(65)$ & 3 \\
6 & $854(162)$ & 9 \\
7 & $890(155)$ & 10 \\
8 & $3032(400)$ & 5 \\
\hline
\end{tabular}




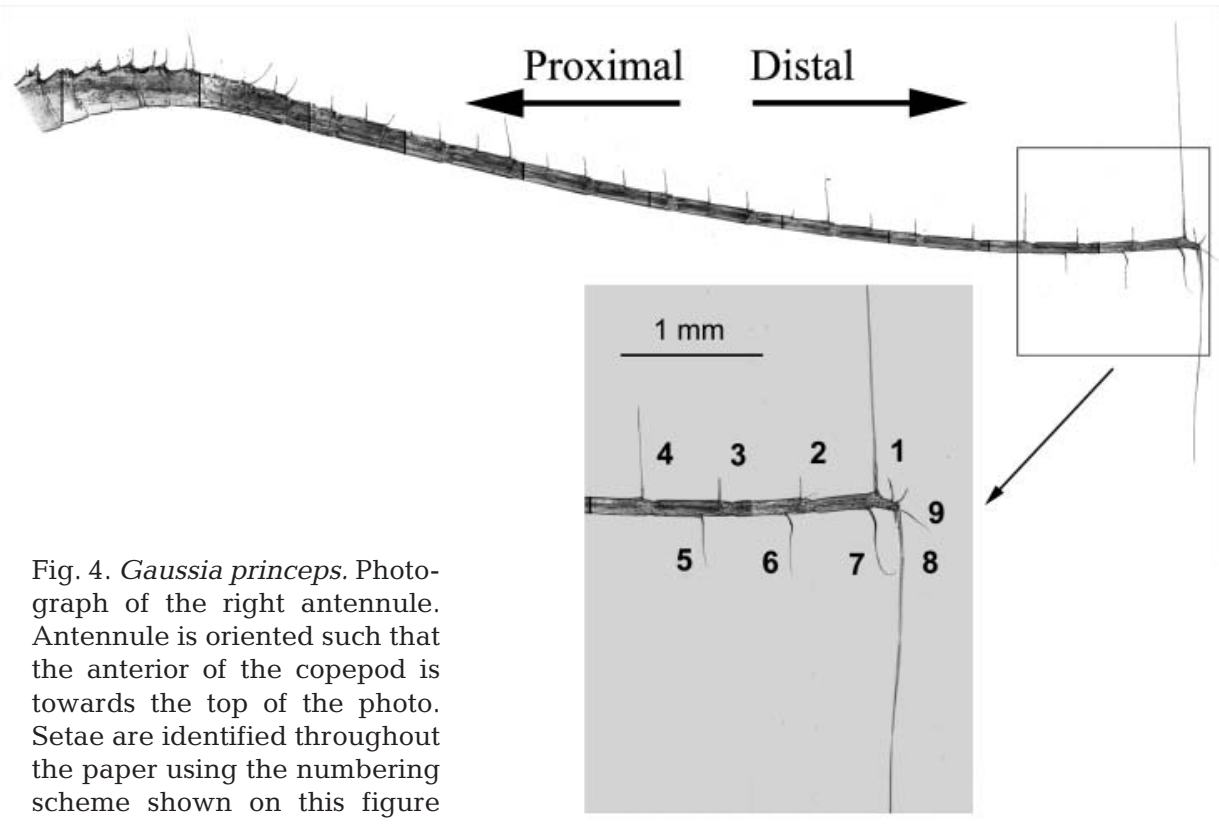

ceps. The physiological response of all the setae tested showed a strong correlation to both angular displacement and angular velocity. However, since angular displacement and angular velocity are tightly correlated in these experiments (Fig. 6), we used angular displacement as our independent variable for further analysis.
Physiological recordings from the tactile stimulation of an individual seta clearly show that the response of different neurons can be discriminated based on their waveform characteristics (i.e. amplitude, rise time, offset slope: Fig. 8A). As such, depending on the orientation of our stimulus, we were able to cause the dis-

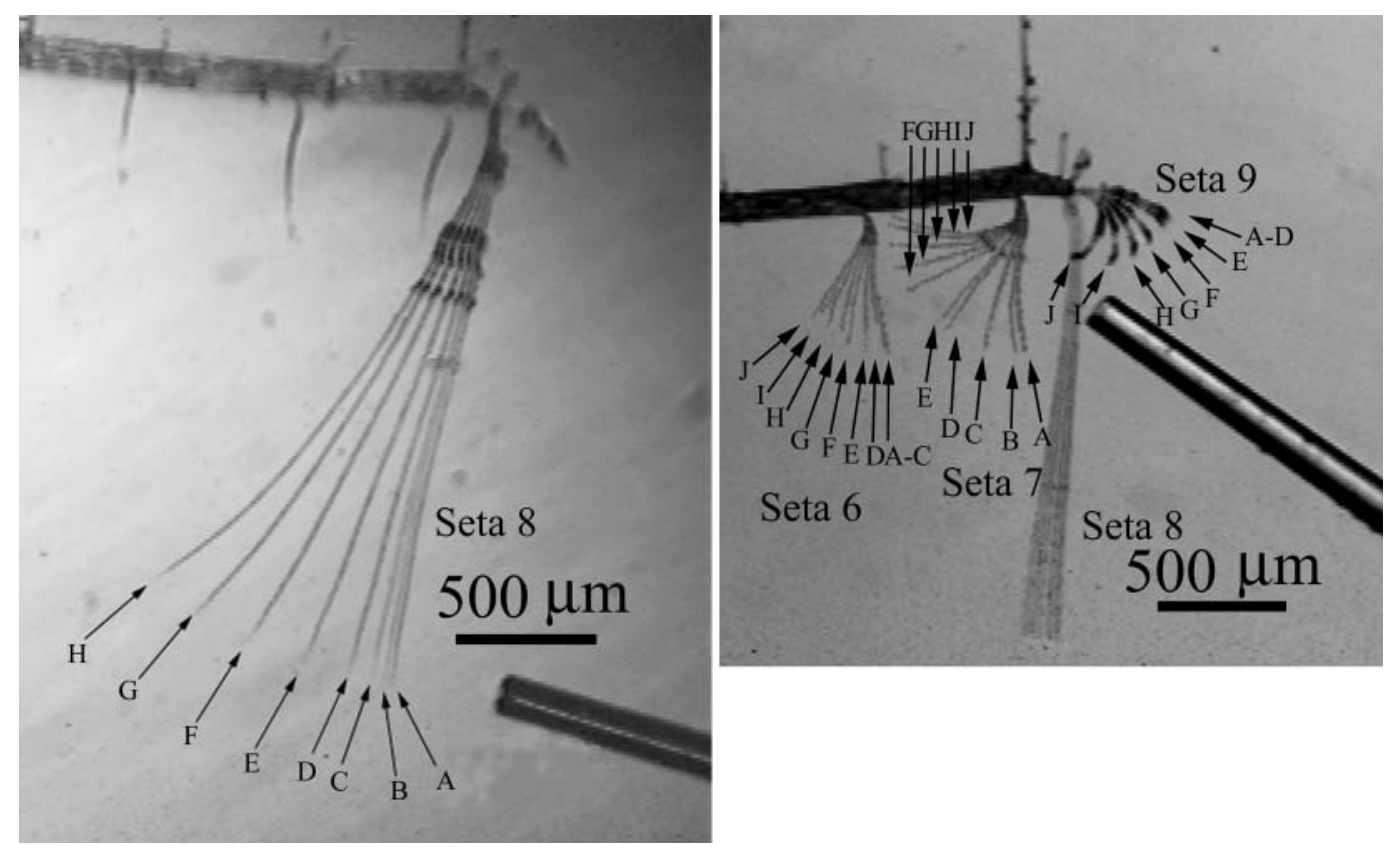

Fig. 5. Gaussia princeps. Maximum angular displacement of distal seta on the antennule. Letters represent the different durations that the solenoid remained opened; each location is the maximum displacement of the seta at that duration (for scale, width of pipette tip is $150 \mu \mathrm{m}$ ). In both photographs, A represents the seta orientation with no stimulation and when the solenoid remained open for $3 \mathrm{~ms}$; each subsequent letter represents a $2 \mathrm{~ms}$ increase in the length of time the solenoid remained open. Left: water jet is aimed at the tip of Seta \#8; right: water jet is aimed at Seta \#7 (note that when Seta \#7 was stimulated in the configuration shown, Setae \#6 and \#9 did not begin to bend until the solenoid remained open for 7 to 9 ms longer) 


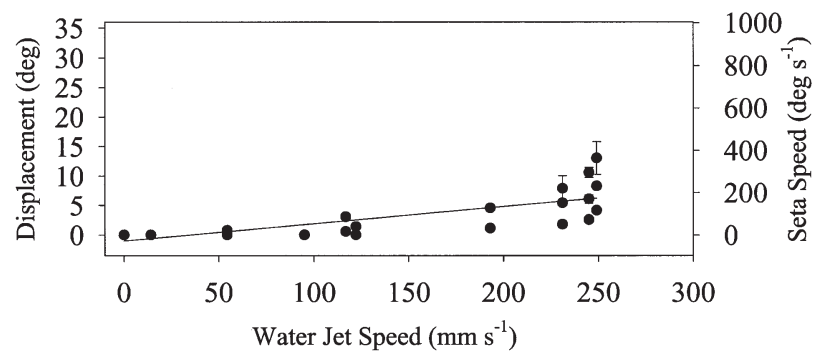

Fig. 6. Gaussia princeps. Maximum angular displacement and average angular velocity of Seta \#8 as a function of water jet speed

placement of 2 different setae from a single pulse of our water jet (Fig. 5). The neural recordings from such events showed the depolarization of the target seta followed by a brief lag period (usually 1 to $10 \mathrm{~ms}$ ) while the water jet traveled to the second seta, and the subsequent depolarization of the second seta (Fig. 8B).

By analyzing the response of individual neurons, we were able to characterize a stimulus intensity-response curve (Fig. 9). In general, once above threshold the physiological response (the number of spikes elicited from the neuron) increased with increasing stimulus strength until a plateau was reached. The increased spike intensity was produced by an increase in the depolarization frequency and the total length of time over which the train of depolarization occurs. For Seta \#8, the physiological threshold was between 0.0 and $0.5^{\circ}$, with saturation occurring at a displacement of approximately $2^{\circ}$.

The physiological sensitivity displayed by an individual seta was not necessarily predictable from an analysis of the physical bending response. The data shows that the longest seta (\#8) has a greater neurological sensitivity to the same angular displacement

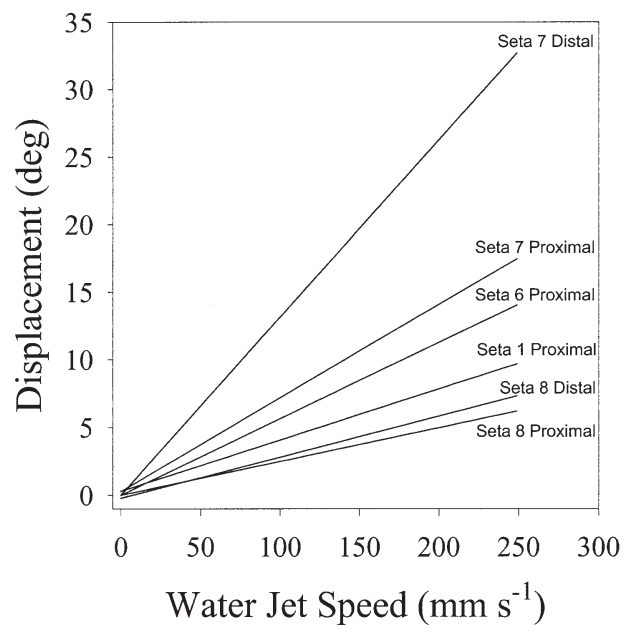

Fig. 7. Gaussia princeps. Maximum angular displacement of different setae as a function of water jet speed, seta tested and the direction of bending. Data was fit to a linear model; statistics are shown in Table 2
Table 2. Gaussia princeps. Tabular results from a linear fit of angular displacement to fluid speed (Fig. 6). Displacement units are in degrees and water speed in $\mathrm{mm} \mathrm{s}^{-1}$. n: number of observations

\begin{tabular}{|lllclr|}
\hline Seta & Direction & Slope & $\mathrm{r}^{2}$ & $\mathrm{p}$ & $\mathrm{n}$ \\
\hline 1 & Proximal & 0.04 & 0.90 & $<0.01$ & 8 \\
6 & Proximal & 0.05 & 0.51 & $<0.001$ & 25 \\
7 & Proximal & 0.07 & 0.41 & $<0.001$ & 34 \\
7 & Distal & 0.13 & 0.51 & $<0.001$ & 18 \\
8 & Proximal & 0.03 & 0.63 & $<0.001$ & 27 \\
8 & Distal & 0.03 & 0.80 & $<0.01$ & 9 \\
\hline
\end{tabular}

than the shorter seta (\#6, \#7; Fig. 10A). Since Seta \#8 has a lower threshold, it reaches physiological saturation at a lower deflection angle than the shorter seta. This is apparent when the angular displacement required for $50 \%$ of the maximum physiological response is compared among setae $\left(\theta_{50}\right.$; Table 3$)$. The halfsaturation constant is lowest in Seta \#8 and increases in the shorter setae, indicating a decreased sensitivity to angular deflections. The poorest sensitivity is for Seta \#7 stimulated distally, requiring approximately 25 times more displacement than Seta \#8 to reach the half-maximal response.

Examining the stimulus intensity-response function expressed in relation to water speed is perhaps more behaviorally relevant, and further emphasizes the disconnection between the physical and physiological response. Seta \#8 is extremely sensitive to bending, particularly in the distal direction, and the low halfsaturation angle of Seta \#8 allows this seta to be relatively sensitive to low water velocity (Fig. 10B,C).

One of the consequences of variation in physiological sensitivity combined with different bending responses is that different seta may code for only a portion of the overall fluid velocity range. For instance, the

Table 3. Gaussia princeps. Physiological response of individual neurons within $200 \mathrm{~ms}$ after stimulation as a function of their angular displacement. Data were fit to a Hill 3-parameter sigmoidal curve (see Eq. 1 in 'Materials and methods'). The half-saturation constant $\theta_{50}$, represents the angular displacement required to elicit $50 \%$ of the maximum physiological response. $\mathrm{n}$ : number of observations. To assure that physiological responses reach saturation, we periodically needed to use water speeds in excess of $250 \mathrm{~mm} \mathrm{~s}^{-1}$. The mechanical stimulus response functions shown in Table 2 are thus a subset of those shown here

\begin{tabular}{|llrccc|}
\hline Seta & Direction & $\theta_{50}$ & $\mathrm{r}^{2}$ & $\mathrm{p}$ & $\mathrm{n}$ \\
\hline 6 & Proximal & 5.6 & 0.64 & $<0.0001$ & 29 \\
7 & Proximal & 5.6 & 0.87 & $<0.0001$ & 40 \\
7 & Distal & 20.3 & 0.70 & $<0.0001$ & 19 \\
8 & Proximal & 4.8 & 0.76 & $<0.0001$ & 35 \\
8 & Distal & 0.9 & 0.92 & $<0.01$ & 9 \\
\hline
\end{tabular}



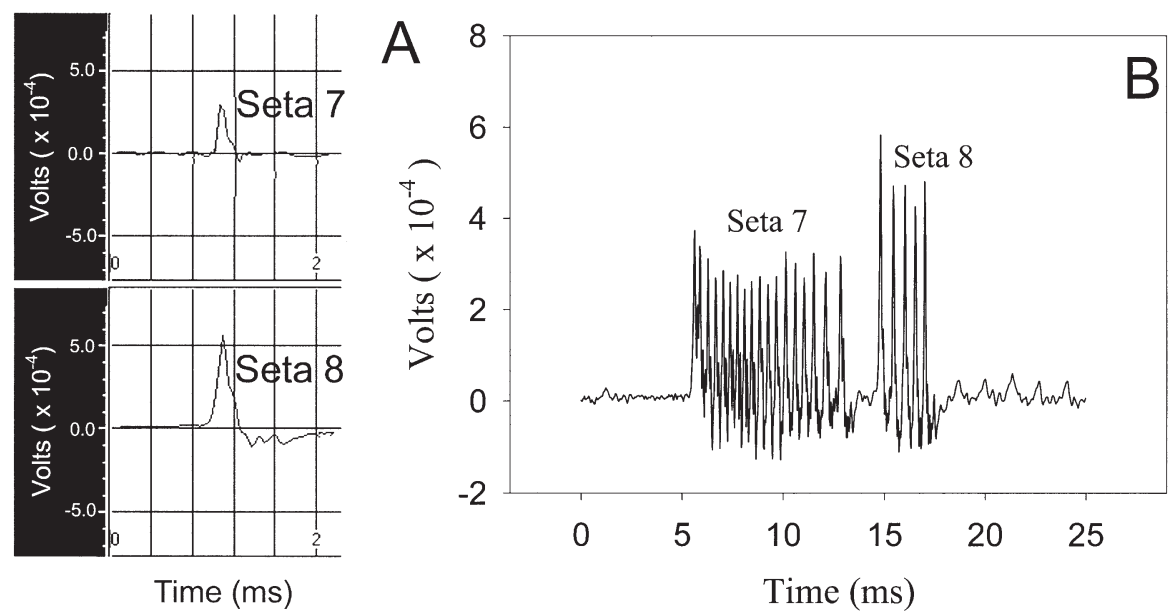

Fig. 8. Gaussia princeps. (A) Depolarization templates created during tactile stimulation of individual seta; template is used to identify the response of individual nerve cells to the water jet when numerous cells concurrently depolarize. (B) Time series of the depolarization events recorded from Setae \#7 and \#8; stimulus was oriented such that Seta \#7 was stimulated to move distally; the expelled water jet first encountered Seta \#7 followed by a slower stimulation of Seta \#8; note the 2 clusters of spikes, the first matched the template of Seta \#7, the second matched Seta \#8 response of Seta \#8 (distal) is extremely steep over a narrow range of low velocity, and reaches saturation at a threshold of about $50 \mathrm{~mm} \mathrm{~s}^{-1}$. In contrast, Seta \#7 shows appreciable response only at velocities above $50 \mathrm{~mm} \mathrm{~s}^{-1}$, with the stimulus intensity response function approximately linear at speeds up to $250 \mathrm{~mm} \mathrm{~s}^{-1}$. Although there is substantial overlap between the sensitivities of the 2 setae, they provide largely complementary information on the water speed.

\section{Directional bending}

We found no physiological evidence that individual seta have multiple innervation. This suggests that if an individual seta were to provide directional information it would need to occur through differential sensitivity to directional stimulation. As previously stated, the directional sensitivity of a given seta reflects changes in both the deflection magnitude to a given fluid velocity (physical response) and the physiological sensitivity to deflection magnitude. When

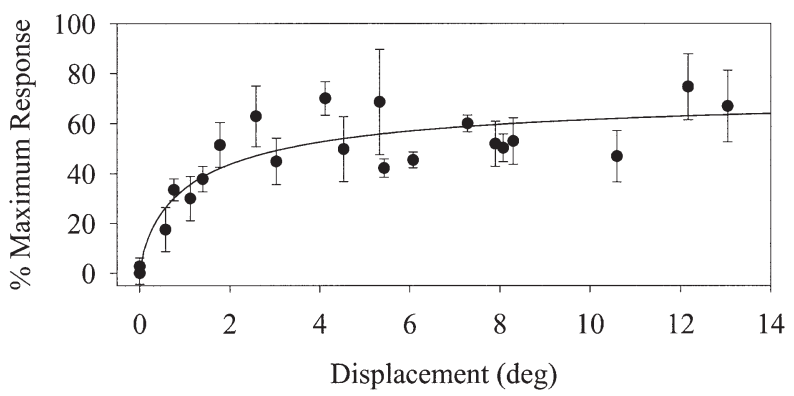

Fig. 9. Gaussia princeps. Physiological response of seta \#8 bent proximally as a function of the water jet speed. In each neuron tested, the data was normalized to the maximum number of spikes recorded
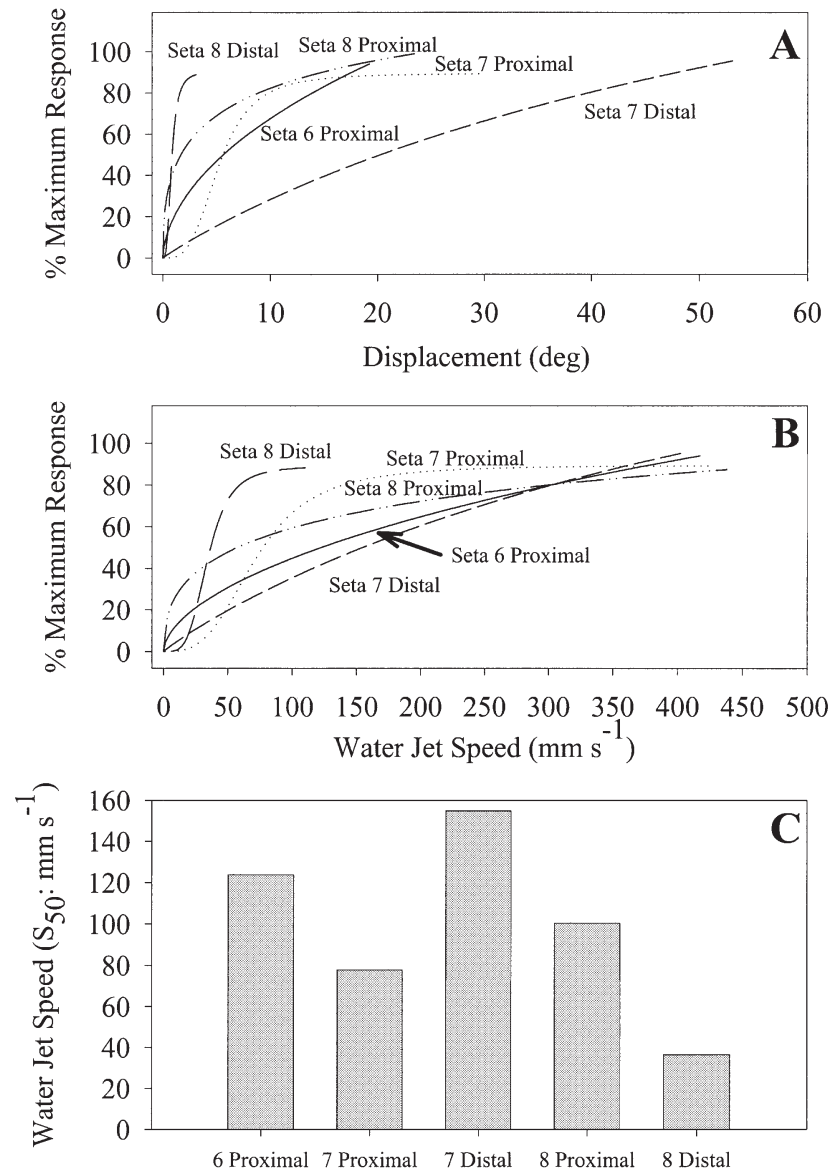

Fig. 10. Gaussia princeps. Physiological response of individual nerves to a fluid mechanical disturbance. (A) Physiological response as a function of the maximum angular displacement of the seta; data was fit to a Hill 3-parameter sigmoid curve (Eq. 1: statistics shown in Table 3); individual lines represent the seta and direction of stimulation. (B) Physiological response of the setae as a function of the water jet speed. (C) Water speed $\mathrm{S}_{50}$ that gives rise to a $50 \%$ maximum physiological response 
excited proximally versus distally, Seta \#8 showed a slight yet significant difference in the $\theta_{50}$ of the stimulus-response function (Table 3). This difference was much more apparent when the physiological response was plotted as a function of the calculated water speed (Fig. 10B). Similarly, Seta \#7, which had a pronounced geniculation in the seta, also had a marked directionality in its physiological response to fluid motion (Table 3). The strong asymmetry in the directional sensitivity of Seta \#7 was again apparent when the physiological response was plotted as a function of water velocity, showing more sensitivity to proximal deflection (Fig. 10B). Note that, like the physiological response in relation to seta length, the neurological directional sensitivity is not easily equated with the physical response of the seta to fluid motion in a particular direction; Seta \#7 was more compliant when subjected to distally directed flow.

\section{DISCUSSION}

Many important behavioral decisions of animals are based on information transmitted through the sensory system. To understand the perceptive abilities, and hence the behavior, of an animal, we must study the mechanisms by which environmental signals are transduced into neurological impulses. It is well established that copepods respond remotely to other organisms, showing a keen ability to spatially locate and attack prey and to avoid capture by potential predators. Previous behavioral observations have shown the importance of mechanoreception in governing different behaviors, yet little work has been done to establish the underlying mechanism of signal transduction. In this study we examined the mechanical and physiological responses of different seta of Gaussia princeps to a controlled fluid mechanical disturbance. The results indicate that a variety of morphological and physiological properties determine the sensitivity of individual seta to fluid speed and direction. The copepod's ability to distinguish spatially heterogeneous fluid signals and locate the source of a fluid disturbance may therefore be contingent upon the ensemble of setal types that comprise the antennule array.

\section{The fluid signal}

How does the copepod detect the magnitude and direction of the fluid disturbance? The size and speed of the source of the fluid disturbance and its distance from the copepods determines the magnitude and structure of the fluid signal. The transduction of the mechanical signal into a physiological signal forms the basis of the copepod's behavioral response. Unfortunately, little is known about the spatial and temporal structure of hydrodynamic signals created by different animals. The fluid signals contain both oscillatory (AC) and unidirectional (DC) components. Previous research has almost exclusively used an oscillating bead to collect behavioral and neurophysiological data from a wide variety of organisms (Horridge \& Boulton 1967, Newbury 1972, Tautz 1979, Gassie et al. 1993, Lenz \& Hartline 1999). Its effects on fluid motion in both the near and far field have been mathematically described (Kalmijn 1988), adding to the relative ease of its use in experimental design. However the high frequencies typically used (100 to $1000 \mathrm{~Hz}$ ), coupled with the extremely small displacements (10 to $1000 \mathrm{~nm}$ ) limit direct comparisons of the results to natural signals. Furthermore, the fluid disturbance radiates from the source, making it difficult to stimulate individual mechanoreceptors on the antennae. Concentrating only on oscillatory stimuli has yielded great insights into mechansensory-mediated behavior and physiology, but other types of stimulus generation methods have begun to reveal potentially important phenomenon not observed using a dipole source (Coombs et al. 1998, Plachta et al. 1999).

In an effort to mimic the wake created by an escaping prey (Yen \& Strickler 1996), or the fluid disturbance of a swimming fish (Breithaupt et al. 1995), we used a small jet to create the fluid disturbance. The solenoid remained open from 3 to $30 \mathrm{~ms}$, giving it an equivalent frequency of 8.3 to $83.3 \mathrm{~Hz}$ and a fluid displacement on the order of millimeters. Visualization of the jet indicates that the expelled fluid moves through the surrounding stationary fluid creating a region of high shear at the leading edge of the jet. As momentum is imparted on the surrounding fluid, through viscous entrainment, the structure of the disturbance becomes very heterogeneous in the distribution of velocity. The resulting speed and fluid structure of the water jet is similar to that found in the toroidal structures created during the escape reaction of large copepods (Yen \& Strickler 1996, Yen 2000). This stimulus can be modeled as a vortex dipole (Voropayev \& Afanasyev 1992) and provides a reproducible and quantifiable level of fluid motion.

A second advantage of using this type of signal over the oscillating bead is that it can be used to excite individual hairs or can be aimed at regions of the antennule to stimulate numerous hairs simultaneously (Fig. 5). This technique allows a comparative analysis of individual setal performance that is unavailable with the dipole source. 


\section{Setal responses}

\section{Physical responses}

A sensory organ with a series of closely spaced flow sensors, such as the antennule of a copepod, provides an ideal structure for detecting fine-scale heterogeneity in a local flow field. All the setae we examined responded to a given fluid velocity with a characteristic angular displacement; however, the magnitude of the displacement was not equal between setae of different lengths. Our results provide empirical evidence that long hairs are physically less sensitive to angular displacement than short hairs, thus supporting the predictions derived from numerical solutions (Devarakonda et al. 1996). All else being equal, Devarakonda et al. calculated that at frequencies between 50 and $500 \mathrm{~Hz}$ (in water), halving the setal length gives rise to a 133 to $300 \%$ increase in angular displacement. The actual degree of displacement is a function of strictly physical features such as the diameter and length of the seta, its mechanical properties (e.g. torsional spring and damping constant; Humphrey et al. 2000), and the viscous drag associated with the medium in which it moves (Devarakonda et al. 1996). In this study, Setae \#7 and \#8 differed in length by nearly a factor of 4 , and yet the smaller seta, Seta \#7, showed only a $200 \%$ increase in maximum displacement. This despite the fact that a proportionally larger area of Seta \#7 was exposed to the stimulus (which should have caused a greater degree of bending). These results suggest that anatomical features such as non-uniform cuticular thickness (Kurbjeweit \& Buchholz 1991) or asymmetry in cross-sectional diameter may differ between setae, thus decreasing the expected difference between setal responses. Shape variation has detectable and predictable effects on the transduction of fluid motion to setal bending in insect wind-receptor hairs (Kumagai et al. 1998), suggesting similar effects may hold in water. Alternately, mechanical features such as degree of setal arcing may affect the relationship between angular deflection and fluid velocity. Due primarily to analytical tractability, most current models of setal motion model the hair as a rigid cylinder of uniform diameter (e.g. Devarakonda et al. 1996, but see Shimozawa et al. 1998), and have generally yielded excellent results when validated against real insect sensilla (Humphrey et al. 2000). In water, however, future efforts to describe the relationship between setal deflection and fluid motion may require models that capture greater biological detail.

\section{Physiological responses}

There are relatively few studies that directly address the characteristics of the setal motion which gives rise to the neurological response in copepods. Previous studies, which have used a vibrating bead as their stimulus, report that Gaussia princeps (Lenz 1993), Euchaeta rimana (Lenz \& Yen 1993) and Pleuromamma xiphias (Lenz \& Yen 1993) can detect fluid displacement as low as 10 to $20 \mathrm{~nm}$ at a corresponding fluid velocity of $\sim 20$ to $40 \mu \mathrm{m} \mathrm{s}^{-1}$. Yen et al. (1992) stimulated individual seta and found similar sensitivities for Labidocera madurae, with the sensitivity to displacement decreasing with decreasing stimulation frequency. To compare sensitivities of different seta, it is helpful to normalize the data to individual setal length and report the displacement characteristics in terms of angular displacement and speed. For example, Yen et al. found that at frequencies above $1000 \mathrm{~Hz}$ the distal seta of $L$. madurae showed a displacement and velocity thresholds of $10 \mathrm{~nm}$ and $\sim 20 \mu \mathrm{m} \mathrm{s}^{-1}$, respectively. For a setal length of $200 \mu \mathrm{m}$ this is equivalent to an angular displacement of $0.003^{\circ}$ and an angular velocity of $6^{\circ} \mathrm{s}^{-1}$ (assuming maximum velocity is reached at half the oscillation cycle; Yen et al. 1992). At frequencies between 250 to $1000 \mathrm{~Hz}$, the threshold displacement for L. madurae remained fairly constant at $\sim 50 \mathrm{~nm}$. This tip displacement corresponds to an angular displacement of $0.01^{\circ}$ and an angular velocity of 5 to $20^{\circ} \mathrm{s}^{-1}$, respectively, which is similar to the sensitivities reported here. At frequencies more likely to occur in nature (<200 Hz: Bleckmann et al. 1991; 10 to $50 \mathrm{~Hz}$ : Yen \& Strickler 1996) and similar to the frequencies used in this study, the threshold displacement required to elicit a neurological response varied inversely with frequency, with the seta becoming 'relatively insensitive, requiring larger displacements and velocities before a [physiological] response is elicited'. We extrapolated their data to the frequencies used in this study to predict that at 85 and $9 \mathrm{~Hz}$ the $200 \mu \mathrm{m}$ seta of $L$. madurae would require a displacement of 0.1 and $2.3^{\circ}$, respectively, and corresponding angular velocities of $17.0^{\circ}$ and $41.4^{\circ}$. These threshold displacements are similar to the $0.5^{\circ}$ displacement threshold found for Seta \#8 (Fig. 10).

These results suggest that at equivalent frequencies the setae of Labidocera madurae requires a greater degree of angular displacement and velocity to elicit a neurological response then those of Gaussia princeps (see Fig. 10A,B). This is consistent with our observations that shorter setae of $G$. princeps are considerably less physiologically sensitive to displacement than larger seta when sensitivity is measured in terms of setal motion. These results also reinforce the importance of using realistic signals. Since the required angular displacement and velocity are functions of stimulation frequencies, the use of high frequencies will overestimate the sensitivity of animals to more natural signals. 
In summary, Devarakonda et al. (1996) predicted, and we have shown empirically, that because smaller setae have a smaller moment of inertia and considerably less drag they are displaced at lower fluid speeds than longer seta. Yet, we also find that shorter setae are less physiologically sensitive than longer setae to angular displacement. Thus, with respect to fluid motion, the sensitivity of the seta depends on the interplay between the relative ease that the seta is displaced (displacement sensitivity) and the threshold angular displacement needed for the neuron to depolarize (physiological sensitivity). This result serves as a warning that determining only the mechanical motion of a sensor may be insufficient for assessing physiological or behavioral sensitivity in copepods. Anatomical studies examining differences in the extension of the dendrites into the shaft of the hairs (e.g. chordadontal versus basal attachment) should offer important insight into the disparity between mechanical and physiological sensitivity.

In Gaussia princeps, the interaction of these 2 features resulted in a long seta relatively sensitive to slow-moving fluid disturbances. The water motion must exceed $20 \mathrm{~mm} \mathrm{~s}^{-1}$ to trigger the physiological response, and act for a sufficient length of time (low frequency) to move the tip of the $3 \mathrm{~mm}$ seta the $110 \mu \mathrm{m}$ needed to reach its $0.5^{\circ}$ threshold angular displacement. G. princeps is a large copepod $(0.8 \mathrm{~cm})$. Its predators are likely to be large fishes and gelatinous zooplankton. As such, the fluid speed created by a predator of this copepod is likely to be well in excess of $25 \mathrm{~mm} \mathrm{~s}^{-1}$. Bleckmann et al. (1991) recorded fluid velocities of $40 \mathrm{~mm} \mathrm{~s}^{-1}$ at a distance of $1 \mathrm{~cm}$ from the tail fin of a cavefish and $50 \mathrm{~mm} \mathrm{~s}^{-1}$ for a $5.5 \mathrm{~cm}$ minnow at a distance of $3 \mathrm{~cm}$. Large fishes up to $22 \mathrm{~cm}$ produce fluid velocities exceeding $60 \mathrm{~cm} \mathrm{~s}^{-1}$ at a distance of $5 \mathrm{~cm}$, which would clearly exceed the threshold of $G$. princeps even at a distance of 7 body lengths. Lauder \& Clark (1984) reported fluid velocities of $25 \mathrm{~mm} \mathrm{~s}^{-1}$ at the mouth of the pumpkin seed sunfish. Furthermore, a review of Fields \& Yen (1997) and Kiørboe et al. (1999) shows that the escape reaction of Pleuromamma xiphias $(6 \mathrm{~mm})$ and Labidocera madurae $(2.5 \mathrm{~mm})$ occurs in fluid velocities of 22 and $27 \mathrm{~mm} \mathrm{~s}^{-1}$, respectively.

In contrast, the shorter setae of Gaussia princeps are displaced at much lower water speeds yet require a large displacement to elicit a neurophysiological response. This combination of high mechanical and low physiological sensitivity produces a sensor that probably responds to higher frequency disturbances. Such signals are produced by escaping copepods, which attain velocities exceeding $600 \mathrm{~mm}$ $\mathrm{s}^{-1}$ (Fields \& Yen 1996) and frequencies in excess of $250 \mathrm{~Hz}$.
Alternatively, acute mechanical and physiological sensitivity may occur in the same seta. Therefore, it is possible that some copepod species may be equipped with small setae that readily move with the prevailing flow and require little motion at the tip to achieve the angular displacements necessary to exceed the physiological threshold. This may explain how the very small seta found on the antennae of Acartia and Temora species provides the sensitivity necessary for detecting and attacking prey that create extremely small fluid disturbances (Jonsson \& Tiselius 1990, Kiørboe et al. 1999).

\section{Discriminating direction}

Accurate attack and escape responses require not only detecting the magnitude of the stimulus, but also its direction. In principle, direction can be discerned from a single seta housing multiple neurons that respond specifically to bending in certain directions. Anatomical observations of Weatherby et al. (1994) for Pleuromamma xiphias suggested multiple innervation of some setae, but we found no physiological evidence of multiple innervation per seta in this study. However, significant asymmetry of the physiological response to proximal versus distal deflections, in combination with directionally specific displacement-velocity curves (e.g. Fig. 6, Seta \#7) produced stimulus-response functions that incorporated directional specificity. In some cases, morphological features were key factors in producing these effects. For example, a cuticular socket that restricts motion of the seta in a particular direction (Wiese 1976, Ball \& Cowan 1977, Yen et al. 1992) can provide a directional bias to the response (Laverack 1976, Tazaki 1977). The elbow located in the lower shaft of Seta \#7 (and \#6; Fig. 5) offers increased rigidity to the hair when bent in the distal compared to the proximal direction, and was therefore partially responsible for the directional sensitivity of this seta. Similar results were found for insect hairs, where the direction of best mobility accurately predicted the directional specificity of the neuronal response (Gnatzy \& Tautz 1980).

However, in Gaussia princeps, it is unlikely that reliable directional signals can be garnered from the response of a single seta, even with a mechanical directional bias. Since the activity of a single neuron depends on both the magnitude and the direction of the stimulus, different combinations of these 2 signal characteristics may lead to a similar neural response (Tautz 1979, Weissburg 1997). The signals from multiple setae, however, provide an excellent mechanism for spatial location. The temporal delay in firing between adjacent seta (Fig. 8B) can be used both to eval- 
uate the speed of the disturbance and the direction. Increased fluid speed would decrease the lag time between the depolarization events and the order that the specific seta fire could distinguish the direction.

The design of mechanoreceptors also may be constrained by operational considerations aside from those pertaining to sensory perception. This suggests that mechanical bias may not always be relevant to directional coding. One potential factor that could influence setal mobility is the necessity for the copepod's body to conform to the demands of locomotion. During the high-velocity escape jumps, the antennules provide propulsive thrust and fold against the body to minimize drag (Strickler 1975, Yen 2000). Setae bend distally during the power stroke, which may account for the preferential bending of Seta \#7. A second factor that may impact on setal mobility is the need to reduce responsiveness to self-generated flow of the feeding currents. This self-generated flow draws the water from the lateral region of the copepod towards its feeding appendages. Setae in the distal region of the antennules are drawn proximally in response to the flow. Thus, the decreased physiological sensitivity to motion in the proximal direction (e.g. Seta \#8), may prevent the fluid motion of the copepod's own feeding current from eliciting a physiological response. The need for decreased sensitivity to proximal fluid motion may be less necessary for setae that have relatively high thresholds, such as Seta \#7. Copepods normally swim at velocities of one to several body lengths $\mathrm{s}^{-1}$ (e.g. Yen 1988, Paffenhoffer \& Lewis 1990, Weissburg et al. 1998) so it is unlikely that Seta \#7 would be greatly stimulated by self-generated motion during normal locomotion.

\section{Discriminating biologically relevant signals from background fluid motion}

Although different mechanical and physiological sensitivities may in some cases reflect constraints ancillary to sensory coding, they may also confer important functional properties onto specific receptor hairs. In spite of its greater resistance to movement, the longer seta (\#8) reached half-saturation at considerably lower fluid speeds than the other setae tested. This suggests that the long setae found on the distal tip function as low-flow detectors. Due to their relatively high viscous drag they are less responsive to highfrequency stimuli and require greater displacement at the tip to reach threshold angular displacement than do shorter seta. The combination of relatively high resistance to movement and extreme sensitivity to small angular displacement results in a steep stimulusresponse function once threshold is achieved. Since the mechanical motion of ambient fluid is small in the low Reynolds number environment in which copepods live, such setal properties allow these animals to be extremely sensitive to specific types of fluid disturbances while providing a filter for background fluid motion.

By peripherally filtering signals using morphologically adapted sensors, the copepod also minimizes the degree of post-processing necessary for signal recognition (Wehner 1987) and, therefore, its reaction time. Since the high sensitivity of the neurological response of Seta \#8 provides maximum detection of fluid signals generated by potential predators located many body lengths away, these long setae appear tuned to lowvelocity displacements (low frequency) similar to those generated by the swimming and feeding motions of fishes. The ability to avoid predation demands high sensitivity, accuracy, and rapidity, all of which are characteristics of the long distal receptor hairs.

The shorter setae (\#6 and \#7), in contrast, respond to high velocity stimuli and require a greater physical displacement than Seta \#8. Maximum response for Seta \#8 (distal) occurs at fluid speeds above $40 \mathrm{~mm} \mathrm{~s}^{-1}$, whereas Seta \#7 (distal) experiences only $20 \%$ of its maximum response at $100 \mathrm{~mm} \mathrm{~s}^{-1}$, and maintains an ability to discriminate among fluid velocities in excess of $400 \mathrm{~mm} \mathrm{~s}^{-1}$. As a result, these sensilla are likely to respond to rapid and powerful fluid displacements similar to those created by an escaping prey.

An important consequence of having adjacent seta that vary in mechanical and physiological properties is that they may have complementary sensitivities. Similar to reports of substantial molecular specificity in chemosensors (Derby \& Atema 1982), it appears that some of the copepod's mechanoreceptors are tuned to detect a specific fluid magnitude range. This is an important feature of the setal array, since the overlap of the stimulus intensity-response functions allows groups of setae to code continuously for fluid velocity even if velocity is outside the range of maximal sensitivity for any one particular seta. Thus, having an ensemble of setae which overlap in sensitivity can provide a copepod with a highly accurate measure of fluid motion over the wide range of flow magnitude seen in the complex flow patterns in natural stimuli (Bleckmann et al. 1991, Yen \& Strickler 1996).

Given the influence of setal morphology on the stimulus transduction process, it is tempting, but unfortunately premature, to speculate on the ecological and evolutionary significance of different sensory morphologies. As a group, the copepods present a spectacular diversity of antennule and setal shapes, sizes, orientations and degree of ornamentation (Huys \& Boxshall 1991). Perhaps when we have arrived at a more systematic and complete survey of the influence of 
setal morphology on the physiological properties of mechanosensory sensilla, it will be possible to predict or explain the behavior of individuals and species based on the appearance of their sensory equipment.

Acknowledgements. We thank Ed Sidel at the Monterey Bay Aquarium Research Institute (MBARI) and the Crew of the RV 'Pt. Sur' for their support and expertise in collecting copepods. We appreciate the critical comments of Dr Jeannette Yen and insights garnered from numerous discussions with Dr Troy Keller. Special thanks to Dr J. A. C. Humphreys for his comments and suggestions. Also we thank 3 anonymous reviewers for their invaluable input. The work was supported by a grant from the National Science Foundation (BioOce/Sensory Biology IBN 981663) to Drs M.J.W. and J.Y.

\section{LITERATURE CITED}

Ball EE, Cowan N (1977) Ultrastructure of the antennal sensilla of Acetes (Crustace, Decapod, Natantia, Sergestidae). Phil Trans R Soc Lond B Biol Sci 277:429-456

Bleckmann H, Breithaupt T, Blickhan R, Tautz J (1991) The time course and frequency content of hydrodynamic events caused by moving fish, frogs and crustaceans. J Comp Physiol A Sens Neural Behav Physiol 168:749-757

Breithaupt T, Ayers J (1996) Visualization and quantitative analysis of biological flow fields using suspended particles. In: Lenz $\mathrm{PH}$, Hartline DK, Purcell JE, Macmillan DL (eds) Zooplankton: sensory ecology and physiology. Gordan \& Breach, Amsterdam, p 117-129

Breithaupt T, Schmitz B, Tautz J (1995) Hydrodynamic orientation in crayfish (Procambarus clarkii) to swimming fish prey. J Comp Physiol A Sens Neural Behav Physiol 177: 481-491

Bundy MH, Paffenhöfer GA (1993) Innervation of copepod antennules investigated using laser scanning confocal microscopy. Mar Ecol Prog Ser 102:1-14

Buskey EJ, Coulter C, Peterson JO, Brown S (1994) Measurements of bioluminescence, distribution and abundance of planktonic bioluminescent organism in the Arabian Sea. In: Biogeochemical processes in the Arabian Sea. Proceedings of US-CIS Arabian Sea Workshop, Sevastopol, Ukraine, p 232-237

Coombs S, Mogdans J, Halstead M, Montgomery J (1998) Transformation of peripheral inputs by the first-order lateral line brainstem nucleus. J Comp Physiol A Sens Neural Behav Physiol 182:609-626

Derby CD, Atema J (1982) The function of chemo- and mechanoreceptors in lobster (Homarus americanus) feeding behavior. J Exp Biol 98:317-327

Devarakonda D, Barth FG, Humphrey JAC (1996) Dynamics of arthropod filiform hairs. IV. Hair motion in air and water. Phil Trans R Soc Lond B Biol Sci 351:933-946

Doall MH, Colin SP, Strickler JR, Yen J (1998) Locating a mate in 3D: the case of Temora longicornis. Philos Trans R Soc Lond B Biol Sci 353:681-689

Drenner RW, McCormas SR (1980) The roles of zooplankter escape ability and fish size selectivity in the selective feeding and impact of planktivorous fish. In: Kerfoot WC (ed) Evolution and ecology of zooplankton communities. University Press of New England, Hanover, p 587-593

Fields DM, Yen J (1993) Outer limits and inner structure: the 3-dimensional flow field of Pleuromamma xiphias (Calanoida: Metridinidae). Bull Mar Sci 53:84-95
Fields DM, Yen J (1996) The escape behavior of Pleuromamma xiphias from a quantified fluid mechanical disturbance. In: Lenz PH, Hartline DK, Purcell JE, Macmillan DL (eds) Zooplankton: sensory ecology and physiology. Gordan \& Breach, Amsterdam, p 560

Fields DM, Yen J (1997) The escape behavior of marine copepods in to a quantified fluid mechanical disturbance. J Plankton Res 19:1289-1304

Friedman MM, Strickler JR (1975) Chemoreceptors and feeding in calanoid copepods (Arthropoda: Crustacea). Proc Natl Acad Sci USA 72:4185-4188

Gassie DV, Lenz PH, Yen J, Hartline DK (1993) Mechanoreception in zooplankton first antennae: electrophysiological techniques. Bull Mar Sci 53:96-105

Gill CW, Crisp DJ (1985) Sensitivity of intact and antennuleamputated copepods to water disturbance. Mar Ecol Prog Ser 21:221-227

Gnatzy W, Tautz J (1980) Ultrastructure and mechanical properties of an insect mechanoreceptor: stimulus-transmitting structures and sensory apparatus of the cercal filiform hairs of Gryllus. Cell Tissue Res 213:441-463

Haury LR, Kenyon DE, Brooks JR (1980) Experimental evaluation of the avoidance reaction of Calanus finmarchicus. J Plankton Res 2:187-202

Horridge GA, Boulton PS (1967) Prey detection by Chaetognatha via a vibration sense. Proc R Soc Biol Sci Ser B 168:413-419

Humphrey JAC, Barth FG, Voss K (2000) The motion sensing hairs of arthropods: using physics to understand sensory ecology and adaptive evolution. In: Barth FG, Schmid A (eds) The ecology of the senses. Springer-Verlag, New York, p 1-21

Huys R, Boxshall GA (1991) Copepod evolution. The Ray Society, London

Jonsson PR, Tiselius P (1990) Feeding behavior, prey detection, and capture efficiency of the copepod Acartia tonsa feeding on planktonic ciliates. Mar Ecol Prog Ser 60:35-44

Kalmijn AJ (1988) Hydrodynamic and acoustic field detection. In: Atema J, Fay RR, Popper AN, Talvolga WN (eds) Sensory biology of aquatic animals. Springer-Verlag, New York, p 83-130

Kiørboe T, Saiz E, Visser AW (1999) Hydrodynamic signal perception in the copepods Acartia tonsa. Mar Ecol Prog Ser 179:97-111

Kumagai T, Shimozawa T, Baba Y (1998) The shape of windreceptor hairs of cricket and cockroach. J Comp Physiol 183:187-192

Kurbjeweit F, Buchholz C (1991) Structure and suspected functions of antennular sensilla and pores of three Arctic copepods Calanus glacialis, Metridia longa, Paraeuchaeta norvegica. Meeresforschung 33:168-182

Lauder GV, Clark BD (1984) Water flow patterns during prey capture by teleost fishes. J Exp Biol 113:143-150

Laverack MS (1976) External proprioceptors. In: Mill PJ (ed) Structure and function of proprioceptors in invertebrates. Chapman \& Hall, London, p 1-63

Lenz PH (1993) Vibration sensitivity in marine copepods. In: Weise K, Gribakin FG, Popov NV, Renninger G (eds) Sensory systems of arthropods. Birkhauser Verlag, Basel, p 423-430

Lenz PH, Hartline DK (1999) Reaction times and force production during escape behavior of a calanoid copepod, Undinula vulgaris. Mar Biol 133:249-258

Lenz PH, Yen J (1993) Distal setal mechanoreceptors of the first antennae of marine copepods. Bull Mar Sci 53: $170-179$ 
Newbury TK (1972) Vibration perception by chaetognaths. Science 236:459-460

Paffenhöfer GA, Lewis KD (1990) Perceptive performance and feeding behavior of calanoid copepods. J Plankton Res 12:933-946

Plachta D, Mogdans J, Bleckmann H (1999) Responses of midbrain lateral line units of the goldfish, Carassuis auratus, to constant amplitude and amplitude-modulated water wave stimuli. J Comp Physiol A 185:405-417

Shimozawa T, Kumagai T, Baba Y (1998) Structural and functional design of the cercal wind-receptor hairs of cricket. J Comp Physiol A 183:171-186

Siaz E, Kiørboe T (1995) Predatory and suspension feeding of the copepod Acartia tonsa in turbulent environments. Mar Ecol Prog Ser 122:147-158

Strickler JR (1975) Swimming of planktonic Cyclops species (Copepod, Crustacea): patterns movements and their control. In: Wu TYT et al. (eds) Swimming and flying in nature, Vol. 2. Plenum, New York, p 599-613

Strickler JR, Bal AK (1973) Setae of the first antennae of the copepod Cyclops scutifer (Sars.): their structure and importance. Proc Natl Acad Sci USA 70:2656-2659

Tazaki K (1977) Nervous responses from mechanosensory hairs on the antennal flagellum in the lobster Homarus gammarus (L.). Mar Behav Physiol 5:1-18

Tautz J (1979) Reception of particle oscillation in a mediuman unorthodox sensory capacity. Naturwissenschaften 66 : 452-461

Voropayev SI, Afanasyev YD (1992) Two-dimensional vortexdipole interactions in a stratified fluid. J Fluid Mech 236: 665-689

Editorial responsibility: Thomas Kiørboe (Contributing Editor), Charlottenlund, Denmark
Weatherby TM, Wong KK, Lenz PH (1994) Fine structure of the distal sensory setae on the first antennae of Pleuromamma xiphias Giesbrecht (Copepoda). J Crustac Biol 14: 670-685

Wehner R (1987) 'Matched filters'-neural models of the external world. J Comp Physiol A 161:511-531

Weise K (1976) Mechanoreception for near-field water displacements in crayfish. J Neurophysiol (Bethesda) 39: 816-833

Weissburg MJ (1997) Chemo- and mechanosensory orientation by crustaceans in laminar and turbulent flows: from odor trails to vortex streets. In: Lehrer M (ed) Orientation and communication in arthropods. Birkhauser Verlag, Basel, p 215-246

Weissburg MJ, Doall MH, Yen J (1998) Following the invisible trail: kinematic analysis of mate-tracking in the copepod Temora longicornis. Phil Trans R Soc Lond B Sens Neural Behav Physiol 353:701-712

Yen J (1988) Directionality and swimming speeds in predator-prey and male-female interactions of Euchaeta rimana, a subtropical marine copepod. Bull Mar Sci 43: 395-403

Yen J (2000) Life in transition: balancing inertial and viscous forces by planktonic copepods. Biol Bull (Woods Hole) 198:213-224

Yen J, Strickler JR (1996) Advertisement and concealment in the plankton: what makes a copepod hydrodynamically conspicuous? Invertebr Biol 115:191-205

Yen J, Lenz PH, Gassie DV, Hartline DK (1992) Mechanoreception in marine copepods: electrophysiological studies of the first antennae. J Plankton Res 14:495-512

Submitted: August 30, 2000; Accepted: April 3, 2001

Proofs received from author(s): February 5, 2002 